\title{
Suporte organizacional, capital psicológico no trabalho e expectativa de futuro
}

\section{em trabalhadores brasileiros}

Organizational support, psychological, capital at work and future expectancy in the Brazilian

\author{
workers
}

Apoyo organizacional, capital psicológico en el trabajo y expectativas futuras en trabajadores

brasileños

\author{
Nilton S. Formiga \\ ORCID: https://orcid.org/0000-0003-4907-9736 \\ Universidade Potiguar, Brasil \\ E-mail: nsformiga@yahoo.com \\ Bruna G. O. Freire \\ ORCID: https://orcid.org/0000-0002-0879-2570 \\ Universidade Potiguar, Brasil \\ E-mail: brunafreireolv@gmail.com \\ Ismael de Mendonça Azevedo \\ ORCID: https://orcid.org/0000-0002-1695-3522 \\ Universidade Potiguar, Brasil \\ E-mail: ismaeldemendonca@bol.com.br \\ Rodrigo L. Nascimento \\ ORCID: https://orcid.org/0000-0002-1291-9992 \\ Universidade Potiguar, Brasil \\ E-mail: rodrigonascimento070187@gmail.com \\ Juliana Bianca Maia Franco \\ ORCID: https://orcid.org/0000-0002-7566-2688 \\ Universidade Potiguar, Brasil \\ E-mail: juliana_franco_@hotmail.com \\ Heitor César Costa Oliveira \\ ORCID: https://orcid.org/ 0000-0003-4778-6613 \\ Universidade Potiguar, Brasil \\ E-mail: cartoriosbrasil@gmail.com \\ Gleyssielle Lira Prochazka \\ ORCID: https://orcid.org/0000-0001-6083-1306 \\ Universidade Potiguar, Brasil \\ E-mail: gleyssielle.lira@gmail.com \\ Sara Ruth Araújo Grangeiro \\ ORCID: https://orcid.org/0000-0001-7446-6020 \\ Universidade Potiguar, Brasil \\ E-mail: sararuthmbc@hotmail.com
}

\begin{abstract}
Resumo
O estudo sobre as organizações e o trabalho tem sugerido novas perspectivas avaliativas referente ao desenvolvimento profissional e de estratégias na gestão de pessoas buscando compreender de forma mais especifica a relação trabalhoemprego-produtividade-saúde. Neste contexto busca-se entender o papel da organização quanto uma dimensão de proteção e segurança social e profissional; desta forma, este estudo tem como objetivo a verificação de um modelo teórico entre o suporte organizacional, capital psicológico positivo e expectativa de futuro. 214 trabalhadores, homens e mulheres, de 19 a 61 anos, de empresas públicas e privadas da cidade do João Pessoa - PB responderam a escala de percepção de suporte organizacional, capital psicológico positivo, expectativa de futuro e caracterização sociodemográfica. Observou-se que o modelo interdependente entre suporte organizacional, capital psicológico e expectativa de futuro, não foi significativo, sugerindo um modelo mediacional, no qual, percepção de suporte organizacional influencia a expectativa de futuro, com o capital psicológico positivo mediando essa relação. De forma geral, destaca-se a importância da organização no investimento emocional (através do capital psicológico) sobre a expectativa de futuro, pois, contribuiria tanto para um melhor funcionamento na vida social e pessoal do funcionário, quanto a possibilidade de uma agendo de educação emocional e funcional na organização.
\end{abstract}

Palavras-chave: Suporte organizacional; Expectativa de futuro; Capital psicológico; Empresas públicas e privadas. 


\begin{abstract}
Studies about ventures and work have suggested new evaluating perspectives related to professional development and strategies on people's management aiming to understand in a more specific way the relation work-job-productivityhealth. In this context, we search to comprehend the venture's role concerning a professional and social protection and safety; thus, this paper aims to verify a theorical model through the organizational support, positive psychological capital and future's expectancy. 214 workers, men and women, aged from 19 to 61 years old, from public and private companies in João Pessoa-PB city answered to the organizational support perception scale, future expectancy and socio-demographical characterization. It has been observed that the independent model through the organizational support, positive psychological capital and future's expectancy was not significant, suggesting a mediational model, in which the organizational support perception influences the future's expectancy, with the psychological capital mediating this relation. In general, the importance of the venture's emotional investment is highlighted (through the psychological capital) about the future's expectancy for it would contribute not only for a better operation in the employee's social and personal life but the possibility of a schedule for a emotional education and functionality in the organization.
\end{abstract}

Keywords: Organizational support; Future's expectancy; Psychological capital; Public and private companies.

\title{
Resumen:
}

El estudio sobre las organizaciones y el trabajo ha sugerido nuevas perspectivas evaluativas sobre el desarrollo profesional y de las estrategias en la gestión de personas, buscando comprender más específicamente la relación trabajo-empleo-productividad-salud. En este contexto, se busca comprender el rol de la organización en términos de protección y seguridad social y profesional; así, este estudio tiene como objetivo verificar un modelo teórico entre el soporte organizacional, capital psicológico positivo y expectativa de futuro. 214 trabajadores, hombres y mujeres, de 19 a 61 años, de empresas públicas y privadas de la ciudad de João Pessoa - PB respondieron la escala de percepción de apoyo organizacional, capital psicológico positivo, expectativa de futuro y caracterización sociodemográfica. Se observó que el modelo de interdependencia entre soporte organizacional, capital psicológico y expectativa de futuro no fue significativo, sugiriendo un modelo mediacional, en el que la percepción de soporte organizacional influye en la expectativa de futuro, con capital psicológico positivo intercediendo esa relación. En general, se destaca la importancia de la organización en la inversión emocional (a través del capital psicológico) sobre la expectativa de futuro, ya que contribuiría tanto para un mejor funcionamiento en la vida social y personal del empleado, como a la posibilidad de una agenda educativa emocional y funcional en la organización.

Palabra clave: Soporte organizacional; Expectativa de futuro; Capital psicológico; Empresas públicas y privadas.

\section{Introdução}

Os estudos com foco na gestão de pessoas e relações humanas no trabalho tem sido abordado desde o clássico pensamento de Elton Mayo, salientando teorias que buscavam apresentar novas perspectivas sobre esta temática. Os pesquisadores tinham o interesse em verificar a influência de variáveis humanas e contextuais (isto é, psicológicas, sociais e psicossociais) na dinâmica organização-trabalho-individuo, visando uma avaliação sobre a produtividade e de saúde laboral. De acordo com Menezes e Gomes (2010), Elton Mayo destacou que os fatores e as configurações físicas e sociais no ambiente de trabalho influenciavam as alterações emocionais e comportamentais significativas nos trabalhadores (Menezes \& Gomes, 2010).

Sendo assim, os estudos nos últimos dez anos sobre a temática tem o interesse na compreensão de variáveis que contemplem explicações muito maiores do que as questões internas da própria organização, busca-se avaliar os eventos profissionais e institucionais que interferem no comportamento do trabalhador no ambiente organizacional salientando os aspectos relacionados à cultura, a motivação, a satisfação, liderança, eficiência, produtividade, valores, atitudes e saúde do trabalhador (Formiga, \& Souza, 2014; Zanelli, Borges-Andrade, \& Bastos, 2004).

Concomitante ao desenvolvimento de estudos e teorias passaram a surgir uma reforma e reestruturação nas normas sociais que regem o trabalho; novas perspectivas e dinâmicas surgem para orientar os contratos psicológicos e físicos que envolvem as relações organização-trabalho-indivíduo, pois as organizações por muito sustentaram uma visão unidirecional relativo ao êxito econômico e de competitividade empresarial, abstendo-se da necessidade de compreender os aspectos humanos que influenciam a atuação quanto ao desempenho e empreendimento do ser profissional (Tractenberg, 1999; Tamayo 
\& Paschoal, 2003; Verstegen, 2011; Scorsolini-Comin, Inocente, \& Miura, 2012; Formiga, Freire, Batista, \& Estevam, 2017; Costa, Costa, \& Cintra, 2018).

Desta maneira, ao longo da trajetória de vida do sujeito, o trabalho pode ser considerado atividade significante, pois funciona como instrumento transformador e integrador por possibilitar a criação e o reconhecimento do trabalhador como indivíduo e ser social (Tolfo, \& Piccinini, 2007), já que as organizações, independentemente de suas características, são empreendimento coletivos (Silva, \& Bastos, 2010). No entanto, dado o contexto contemporâneo de transformações sóciopolíticas, a relação organização-trabalho-individuo tem se apresentado bastante enfraquecida e com perspectiva de futuro incerto. Fato esse, que se deve a transformação do mercado em um ambiente complexo e instável, influenciando a atuação e desempenho dos trabalhadores, tornando com isso, um ambiente inóspito e hostil (Ng \& Parry, 2016).

Com a transformação do mercado, estudos buscam investigar a área de gestão de pessoas, tendo interesse na compreensão de temas que envolvem a tríade organização-trabalho-indivíduo e apresentar explicações e intervenções que auxiliem na manutenção de uma política de empregabilidade mais valorosa e competitiva no contexto organizacional e social, integrando os objetivos organizacionais e profissionais (Formiga \& Souza, 2014; Puente-Palacios \& Peixoto, 2015). Especialmente, quanto a compreensão dos aspectos intrínsecos do trabalhador, relacionados à emoção, sentimentos, prazer, satisfação, sonhos, criatividade e a intuição que constitui e influência na atuação do ser profissional (Moreno, 2002).

No Brasil, estudiosos têm utilizado a teoria do suporte organizacional como uma das medidas para explicar o comportamento organizacional e sua influência na qualidade de vida e bem-estar do trabalhador (Siqueira, 2009). Esse constructo busca avaliar a percepção do profissional acerca das ações de reconhecimento e valorização que a organização possui em relação à atuação profissional, a medida de suporte organizacional possibilita verificar os principais impactos das práticas cotidianas organizacionais na atuação do trabalhador na empresa, quer seja na esfera pública ou privada, gerando por vezes a sócio cognitivamente, que é uma percepção no profissional de que a empresa o apoia (Jawahar \& Hemmasi, 2006; Tamayo \& Abbad, 2006; Dawley, Andrews, \& Bucklew, 2008; Richardson, Yang, Vandenberg, DeJoy, \& Wilson, 2008; Byrne, \& Hochwarter, 2008; Fleury, Formiga, Souza, \& Souza, 2017).

O suporte organizacional no ambiente corporativo pode ser percebido a partir de ações de reconhecimento e valorização relacionadas a feedbacks de aprovação, elogios, recursos financeiros ou formas de representação social (OliveiraCastro; Pilati, \& Borges-Andrade, 1999). Além do mais, pode se relacionar com variáveis associadas à percepção do significado do trabalho e criatividade dos funcionários (Akgunduz, Alkan, \& Gök, 2018; Ibrahim, Isa, \& Shahbudin, 2016), geração de empregos, desgaste e satisfação (Martinez \& Paraguay, 2003; Ascenção, 2009). Bem como, interferir para a redução dos sintomas da síndrome de burnout (Altinoz, Cop, Cakiroglu, \& Altinoz, 2016), e na redução de conflitos trabalhofamília (Ibrahim \& Marri, 2015); gestão do conhecimento (Burmeister, \& Deller, 2016); processo de mudança (Ferreira, Cardoso, \& Braun, 2018; Al-Hussami, Hammad, \& Alsoleihat, 2018) e comprometimento organizacional (Fernandes, Siqueira, \& Vieira, 2014; Celep \& Yilmazturk, 2012).

O suporte pode ser considerado instrumento mediador na relação organização-trabalho-individuo, já que representa uma espécie de contrato psicológico em que ocorre uma troca percebida pelo trabalhador como retribuição ao esforço despendido em sua atividade laboral na organização (Eisenberger et al., 1986; Brandão, Borges-Andrade, \& Guimarães, 2012). Sendo assim, o suporte está no resultado da subtração entre as expectativas e os benefícios mútuos estabelecidos entre o trabalhador e a organização, que tem por consequência a motivação dos profissionais na busca constante de melhorias para o seu próprio desempenho nas atividades, quer seja através da aprendizagem ou capacitação profissional (Oliveira-Castro, Pilati, \& Borges-Andrade, 1999; Chong, White, \& Prybutok, 2001; Paschoal, 2008; Ng, 2017).

Além disso, os estudos desenvolvidos por Cooper-Hakim e Viswesvaran (2005) e Ströbel, Maier e Woratschek (2018) apontam que o constructo suporte organizacional apresenta relação significativa no tocante à redução dos níveis de 
rotatividade, pois o sujeito diante da sua própria identificação das formas de reconhecimento, valorização, e apoio que a organização possui para com sua atuação, constrói uma crença positiva acerca da imagem da empresa, e consequentemente acaba não tendo interesse em mudar de organização (Islam, Ali \& Ahmed, 2018).

A baixa rotatividade, por sua vez, se apresenta em pesquisas recentes quanto ao suporte organizacional e a expectativa de futuro, pois intervém sobre a percepção de esperança e otimismo na vida do indivíduo, garantindo a confiança de uma carreira de longo prazo na organização, que por consequência influencia na atuação e desempenho profissional (Formiga, Freire, \& Estevam, 2018).

É importante reforçar que a expectativa de futuro de um profissional, em uma dada empresa, pode intervir em variáveis relacionadas à cidadania organizacional, desempenho e qualidade da atuação destes trabalhadores, pois a percepção do indivíduo quanto ao longo prazo pode estimular o sentimento de pertencimento à organização, que porventura influencia no comportamento assertivo e dedicado à atuação individual perante a missão, visão e aos objetivos organizacionais (Joireman, Daniels, George-Falvy, \& Kamdar, 2006; Graso \& Probst, 2012; Lee, Yun, \& Kim, 2017).

No contexto contemporâneo, pensar sobre o futuro profissional possibilita ao indivíduo a experimentação de afetos, cultivo de preferências ou desejos, expressão de expectativas, percepções ou crenças sobre a posterioridade, contudo, este planejamento quanto ao futuro tem sido cada vez mais uma atitude de profissionais na busca por refletir sobre a sua carreira no mercado empresarial em que atua (Vasconcelos, 2015).

O trabalhador desenvolve atitudes profissionais refletidas em maior segurança e confiança, já que a construção das expectativas e convicções de futuro sobre o próprio desempenho desenvolve a integração e sintonia das suas próprias aprovações assim como das pessoas que estão ao seu redor (Buscacio \& Soares, 2017).

As pessoas agem com base em determinado grau de previsão quanto ao futuro (Souza, Pereira, Funck, \& Formiga, 2013), desta forma, esta prática pessoal se reflete no âmbito do ambiente empresarial, onde definir perspectivas para o futuro da organização pode ser considerada uma maneira eficiente de moldar as experiências vivenciadas no passado e presente, o que incentiva práticas determinantes para a mudança organizacional, pois desenvolver uma percepção de futuro incentiva a criação de profecias autorrealizáveis, promovendo tomadas de decisões mais assertivas (Drucker, 1959; Weick, 1979; Ford, 2002).

Assim, para Vasconcellos e Neiva (2017) se torna útil monitorar as expectativas de futuro organizacional a fim de mitigar possíveis efeitos negativos de expectativas desfavoráveis, ao tempo que se pode potencializar eventuais efeitos positivos de expectativas favoráveis, assim profissionais cada vez mais os profissionais ficam comprometidos, satisfeitos, otimistas e menos propensos a sair da organização (Mossholder, Settoon, Harris, \& Armenakis, 1995; Hubbard \& Purcell, 2001; Margolis \& Hansen, 2003; Chiu, 2002; Zhou, Li, \& Zhou, 2004; Chiu, Hui, \& Lai, 2007).

Por vezes, a expectativa de futuro no ambiente organizacional acaba sendo confundida com orientação profissional para o futuro, já que para alguns sujeitos indicam os mesmos objetivos. Portanto, é necessário compreender as principais diferenças acerca das perspectivas, pois expectativa de futuro envolve as percepções de longo-prazo do sujeito formuladas a partir dos sentimentos e emoções que o indivíduo desenvolve com base nos objetivos e metas que está fadado a alcançar, seja de cunho pessoal, profissional ou social, enquanto que a segunda se propõe a práticas atitudinais relacionadas ao realismo, planejamento e controle, indicando que o indivíduo traça ações no presente com vistas a atingir determinado resultado no futuro (Nurmi, 2005; Seginer, 2009). Ou seja, a expectativa de futuro compreende as percepções do sujeito sobre o que está por vir, enquanto a orientação profissional para o futuro está atrelada a definição e planejamento de práticas no presente visando o alcance de um objetivo maior no futuro.

A expectativa de futuro, por sua vez, se caracteriza como uma crença de que existe um futuro que já está anunciado para o indivíduo, propondo que a imagem que se faz do futuro termina por influenciar o comportamento do sujeito e grupos no presente (Seginer, 2009; Formiga, Fleury, \& Souza, 2015). Ou seja, quanto maior a perspectiva (ou a crença) do futuro, maior 
a chance de tomar decisões mais acertadas no presente. De fato, aquilo que é visto como possibilidade no futuro termina por influenciar as decisões que são tomadas no presente, seja pelo cidadão comum, por empresas e até mesmo por nações (Günther, \& Günther, 1998; Robbins, \& Bryan, 2004; Aspinwall, 2006; Sulimani-Aidan \& Benbenishty, 2011).

A trajetória histórica acerca dos estudos sobre expectativa de futuro envolve diversas perspectivas. Considerado como o pesquisador pioneiro nesta temática, Omar Delgado, Souza e Formiga (2005) define que a expectativa de futuro pode ser compreendida por meio de duas abordagens: a primeira está relacionada a formação dos vínculos que a expectativa de futuro incentiva, já que promove o desenvolvimento da confiança nos outros a partir das crenças sobre a predição, controle das pessoas e da natureza. Já a segunda abordagem procura associar a expectativa de futuro com traços de personalidade e atitudes positivas do sujeito, que conseguem ser observadas e identificadas no meio social.

Além disso, Cárdenas, Loving e Lagunes (2001) retratam que a expectativa de futuro pode ser explicada e influenciada pelos preceitos da educação familiar, pois os ensinamentos vivenciados no laço familiar podem impactar o comportamento do sujeito, que por consequência, gera no individuo atitudes formuladas com base nos estímulos recebidos. Neste ínterim, o indivíduo que recebe ao longo da vida apenas estímulos negativos e de reprovação, pode fazer com que o processo de reconhecimento acerca do futuro seja repleto de sentimentos de desaprovação e desdém consigo, enquanto o sujeito que vivencia a educação baseada em estímulos positivos, apresenta uma maior chance de desenvolver expectativas mais otimistas e confiantes acerca do futuro (Schmidt, 2008).

Dessa forma, como forma de compreender e aprofundar o estudo acerca das áreas cientificas que possam explicar sobre a evolução do comportamento assertivo do sujeito no meio social, torna-se necessário para o presente estudo resgatar a importância da Psicologia Positiva, pois, tal perspectiva na área da ciência psicológica, foca nos principais benefícios e impactos do comportamento baseado em práticas positivas na vida do indivíduo em detrimento da exploração dos prejuízos que as condutas humanas e sociais venham causar (Ujhelyi, Carson, \& Holland, 2016).

Neste sentido, Linley, Govindji e West (2007), consideram que a Psicologia Positiva pode ser considerada área fundamental para o desenvolvimento dos sujeitos em diversas áreas do conhecimento como humana, social e da saúde, por buscar incentivar nas pessoas uma reflexão sobre o seu melhor estado de si, de maneira que possa identificar o que é certo, o que está funcionando, o que é importante, para desta forma potencializar os sentimentos e emoções positivas de maneira que pode auxiliar ao indivíduo a se tornar cada vez mais saudável, capaz de enfrentar os conflitos e desafios presentes no dia a dia, com otimismo e entusiasmo (Seligman \& Csikszentmihalyi, 2000).

No âmbito organizacional, a Psicologia Positiva tem como objetivo contribuir para o desenvolvimento do bem-estar no trabalho e na organização, garantindo profissionais mais engajados, confiantes e saudáveis. No entanto, torna-se necessário que a cultura do ambiente organizacional seja formulada com base em intervenções positivas de reconhecimento e valorização de forma que possibilite aos profissionais desenvolverem um comportamento assertivo espontâneo, para garantir satisfação e sintonia entre os objetivos da empresa e do trabalhador (Suchman, Sluyter, \& Williamson, 2011; Ouweneel, Blanc, \& Schaufeli, 2013).

Estes aspectos fazem com que os estudos na área da psicologia organizacional e do trabalho se interessem em avaliar o comportamento organizacional positivo (COP), que é caracterizado pela presença de quatro variáveis básicas: a) esperança; (b) auto-eficácia; (c) resiliência; e (d) otimismo. A combinação destas características gera no sujeito a construção de um estado mental positivo definido como: capital psicológico ou psycap, a partir do qual, o sujeito com confiança, otimismo, esperança e resiliência consegue definir as ações necessárias para obter sucesso no presente e no futuro, baseado em atitudes de progressão, recuperação e resistência para lidar com os problemas e desafios do dia a dia (Luthans, 2002; Luthans, Avolio, Avey, \& Norman, 2007; Luthans, Youssef, \& Avolio 2007). 
É válido informar que tal perspectiva de estudo evoluiu para fatores que estão além dos clássicos de avaliação na área da organização e do trabalho, como por exemplo: motivação e estresse. Atualmente, enfoca pesquisas quanto à intervenção em variáveis sobre otimismo, criatividade, humildade, perdão, gratidão, coragem, autocontrole, sinceridade etc. Não obstante, parte do pressuposto que estes aspectos poderão influenciar em fatores atitudinais relacionados ao comprometimento, empoderamento, absenteísmo, engajamento, satisfação e desempenho (Avey, Patera \& West, 2006; Avey, Hughes, Norman, \& Luthans, 2008a; Avey, Luthans, \& Mhatre, 2008b; Avey, Luthans, \& Jensen, 2009; Han, Brooks, Kakabadse, Peng, \& Zhu, 2012).

Pesquisas recentes sobre capital psicológico destacam em seu contexto que a variável possui relações significativas com comprometimento de carreira e bem-estar (Singhal \& Rastogi, 2018), performance profissional (Tüzün, Çetin, \& Basim, 2018), liderança e engajamento no trabalho (Li, Castaño, \& Li, 2018; Kang \& Busser, 2018; Karatepe \& Karadas, 2015), desempenho no crowdfunding (Anglin, Curto, Drover, Stevenson, McKenny, \& Allison, 2018), liderança compartilhada, criatividade e comprometimento (Wu, \& Chen, 2018), redução nos níveis da síndrome de burnout e aumento do desempenho no trabalho (Rehman, Qingren, Latif, \& Iqbal, 2017), satisfação no trabalho (Badran \& Youssef-Morgan, 2015), desempenho percebido, intenção de rotatividade e bem-estar (Choi \& Lee, 2014), entre outras mais.

De acordo com Ahmad e Zafar (2018) a promoção de um ambiente de trabalho baseado na confiança e respeito mútuo pode incentivar a construção de valores positivos dos trabalhadores, pois a percepção de um ambiente organizacional democrático e com oferecimento de insights significativos influenciam no comprometimento, criatividade e desempenho do trabalhador (Han \& Garg, 2018). Desta forma, a presente pesquisa tem como objetivo verificar a influência do suporte organizacional sobre o capital psicológico e a expectativa de futuro dos trabalhadores brasileiros de organizações públicas e privadas.

O interesse neste tema para área da psicologia organizacional e na administração se deve não apenas pela quantidade de estudos que focam na importância do capital psicológico no trabalho, mas também pela recente consulta nos sites de busca a partir das associações das palavras-chaves: suporte organizacional, capital psicológico, expectativa de futuro; trabalhadores e psycap, expectativa, suporte, organizações e sociedade, suporte, organização, comportamento positivo, expectativa e trabalho, as quais não permitiram encontrar estudos que contemplassem a relação entre as três variáveis aqui propostas.

Com isso, o estudo justifica-se tanto pela sua originalidade para o viés acadêmico e profissional, quanto por sua condição de aplicabilidade na área do recurso humano; desta maneira, hipoteticamente, espera-se que o suporte organizacional se relacione positivamente com o capital psicológico e com a expectativa de futuro, e, que estas últimas variáveis também se relacionem entre si.

\section{Metodologia}

\section{Amostra}

O presente estudo se embasou numa abordagem quantitativa, considerando apenas os participantes que estavam empregados e ativos no seu setor de trabalho, CLT, devendo ter 6 horas ou mais de trabalho e com idade acima de 18 anos. Tratou-se de uma amostra não probabilística, considerando somente a pessoa que, consultada, dispusera-se a colaborar respondendo o questionário apresentado. Com isso, uma amostra de 214 sujeitos, a qual, foi avaliada no pacote estatístico GPower 3.1, com base em critérios estatísticos [probabilidade de 95\% ( $\mathrm{p}<0,05)$, magnitude do efeito amostral $(\mathrm{r} \geq 0,30)$ e um padrão de poder hipotético $(\pi \geq 0,80)$ ], observando que o ' $n$ ' em questão foi suficiente ( $t \geq 1,98 ; \pi \geq 0,97 ; \mathrm{p}<0,05$ ). 
Desta forma, na amostra, os respondentes apresentaram idade variando de 19 a 61 anos, $32 \%$ pertenciam a empresas públicas e $68 \%$ de empresas privadas da cidade do João Pessoa - PB, 57\% eram do sexo feminino, 25\% tinham 2 anos de trabalhos e $38 \%$ ganhavam acima de $2.500,00$ reais.

\section{Instrumentos}

Os sujeitos responderam os seguintes instrumentos:

Escala de Percepção de Suporte Organizacional - EPSO: trata-se de uma medida, é composto por 9 itens, foi originalmente desenvolvida por Eisenberger et al. (1986) e validada para população brasileira por Siqueira (1995). A presente escala é voltada para a avaliação da percepção do sujeito no que se diz respeito o quanto a empresa se preocupa com o bemestar do empregado. No estudo de Siqueira (1995), o instrumento apresentou um alfa de 0,86, revelando uma consistência interna na mensuração do construto. O sujeito deveria indicar sua resposta, marcando com um $\mathbf{X}$ ou um círculo, em uma escala de sete pontos que variava de $1=$ discordo totalmente, $2=$ discordo moderadamente, $3=$ discordo levemente, $4=$ nem concordo, nem discordo, $5=$ concordo levemente, $6=$ concordo moderadamente e $7=$ concordo totalmente.

No Brasil, um estudo desenvolvido por Formiga, Fleury e Souza (2014), utilizando a análise fatorial confirmatória, com o objetivo de avaliar a organização da estrutura fatorial da EPSO com amostras de trabalhadores de organizações públicas e privadas, observaram indicadores psicométricos confiáveis $\left(\chi^{2} / \mathrm{gl}=1,42, \mathrm{RMR}=0,02, \mathrm{GFI}=0,99, \mathrm{AGFI}=0,97, \mathrm{CFI}=0,99\right.$, TLI = 0,99 e RMSEA = 0,03), os quais, confirmam a estrutura unifatorial da EPSO, previamente, proposta por Siqueira (1995).

Escala de capital psicológico positivo no trabalho (ECPP) - Trata-se de um instrumento elaborado por Luthans, Youssef e Avolio (2007); neste estudo utilizou-se a forma reduzida para 12 itens, desenvolvida por Viseu et al. (2012) para o contexto português. A escala é composta por 12 itens que descrevem para os respondetes, um estado psicológico positivo com o objetivo para enfrentar e empregar o esforço necessário para alcançar sucesso em tarefas desafiadorasa. De acordo com os autores, a escala é formada por quatro fatores: otimismo ( 2 itens), resiliência (3 itens), autoeficácia (3 itens) e esperança (4 itens). O sujeito deverá responder numa escala tipo Likert, de seis pontos, o grau de concordância com cada itens (1 - Discordo fortemente; 2 - Discordo; 3 - Discordo um pouco; 4 - Concordo um pouco; 5 - Concordo; e 6 - Concordo fortemente)

Escala de expectativa de futuro - EEF: Trata-se de uma escala adaptada e validada por Souza, Pereira, Funk e Formiga (2013) para o contexto brasileiro, a qual, tanto na análise exploratória quanto na confirmatória, revelou indicadores psicométricos satisfatórios e que estavam de acordo com o padrão psicométrico exigido pela literatura estatística; a mesma é composta por 18 itens distribuídos em três fatores: melhores condições da sociedade (MCS) [por exemplo, estarei muito bem de saúde; Serei orgulhoso de mim mesmo por ter lutado e vencido na vida; etc.]; sucesso profissional e financeiro (SPF) [por exemplo, estarei realizado profissionalmente; Terei dificuldades em obter um emprego digno, etc.] e realização pessoal (RP) [por exemplo, as pessoas terão maior possibilidade de realizar seus sonhos, Terei a certeza de que minha vida é um fracasso, etc.]. A presente escala tinha o objetivo de avaliar quais as expectativas que o respondente tem em relação aos planos, aspirações e medos em relação a vários domínios da vida em breve ou distante; este deveria indicar a sua resposta em uma escala do tipo Likert variando de $1=$ totalmente ruim, $2=$ em parte será ruim, $3=$ Nem será ruim e nem será bom, $4=$ Em parte será bom, 5 = totalmente bom.

É preciso salientar que a presente escala ainda não havia sido administrada em amostra de trabalhadores de instituições públicas e privadas; no estudo desenvolvido por Formiga, Fleury e Souza (2015) esta escala apresentou uma organização trifatorial com indicadores psicométricos que garantiram a sua consistência $(\chi 2 / \mathrm{gl}=1.42, \mathrm{RMR}=0.02$, GFI $=$ 0.99, $\mathrm{AGFI}=0.98, \mathrm{CFI}=0.99, \mathrm{TLI}=0.98, \mathrm{RMSEA}(90 \% \mathrm{IC})=0.03(0.010 .04), \mathrm{CAIC}=889.15 \mathrm{e} \mathrm{ECVI}=0.55)$.

Tendo em vista a orientação fatorial do respectivo construto, optou-se por verificar, através da análise fatorial confirmatória, a adequabilidade teórica e psicométrica desta medida na respectiva amostra; esta, revelou indicadores 
confiáveis, condição que garantiu a medida da expectativa de futuro em trabalhadores $(\chi 2 / \mathrm{gl}=2,57, \mathrm{GFI}=0.95, \mathrm{AGFI}=0.97$, $\mathrm{CFI}=0.98, \mathrm{TLI}=0.97, \mathrm{RMSEA}(90 \% \mathrm{IC})=0.05)$.

Caracterização sociodemográfica. Foram elaboradas perguntas que contribuíram para caracterizar os participantes deste estudo (por exemplo, sexo, idade, tempo de trabalho e empresa em que trabalha), bem como, realizar um controle estatístico de algum atributo que possa interferir diretamente nos seus resultados.

\section{Procedimentos}

Todos os procedimentos adotados nesta pesquisa seguiram as orientações previstas na Resolução 196/96 do CNS e da Resolução 016/2012 do Conselho Federal de e Psicologia. O mesmo, foi submetido ao CONEP e aprovado pelo sob o protocolo de pesquisa $\mathrm{n}^{\mathrm{o}}$ 15827919.0.0000.5296

\section{Administração}

Para a aplicação do instrumento, os responsáveis pela coleta dos dados visitaram as empresas e instituições de ensino superior na cidade de João Pessoa-PB; nas empresas falava-se diretamente com os diretores ou responsáveis diretos dos funcionários e nas instituições com os coordenadores e professores responsáveis pela aula, procurando obter sua autorização para ocupar um período do seu trabalho ou aula aplicar os questionários. Sendo autorizado, os responsáveis foram contatados, expondo sumariamente os objetivos da pesquisa, solicitando sua participação voluntária. Foi-lhes dito que não havia resposta certa ou errada e que mesmo necessitando uma resposta individual, estes não deveriam se ver obrigados em respondê-los podendo desistir a qual momento seja quanto tivesse o instrumento em suas mãos ou ao iniciar sua leitura, ou outra eventual condição; em qualquer um desses eventos, não haveria problema da desistência do respondente.

A todos era assegurado o anonimato das suas respostas e que estas seriam tratadas em seu conjunto estatisticamente; apesar do questionário ser autoaplicável, contando com as instruções necessárias para que possam ser respondidos, os colaboradores estiveram presentes durante toda a aplicação para retirar eventuais dúvidas ou realizar esclarecimentos que se fizessem indispensáveis, não interferindo na lógica e compreensão das respostas dos respondentes. Em cada empresa um único aplicador, previamente treinado, esteve presente no local, apresentando os instrumentos, solucionando eventuais dúvidas e conferindo a qualidade geral das respostas emitidas pelos respondentes.

Com base numa análise estatística mais criteriosa e rigorosa, testou-se o modelo teórico a partir da modelagem de equação estrutural, considerando índices estatísticos que permitem avaliar a qualidade de ajuste do modelo proposto (Van De Vijver \& Leung, 1997; Hair, Tatham, Anderson, \& Black, 2005; Byrne, 2012), por exemplo: ( $\chi^{2} / g .1$, GFI, AGFI, RMSEA, CFI, NFI. Também, foi realizado o teste de Sobel $(z)$, o qual, o tem o objetivo de avaliar o efeito da variável mediacional entras as variáveis do estudo.

\section{Resultados}

Após a coleta de dados, procurou-se atender ao objetivo principal do estudo (pretendeu-se verificar um modelo teórico, no qual, o suporte organizacional, capital psicológico no trabalho e expectativa de futuro seriam construtos interrelacionados numa amostra de trabalhadores). A partir da análise e modelagem de equação estrutural, considerando um modelo recursivo de equações estruturais, realizaram-se cálculos que tinham a função de comprovar o modelo hipotetizado.

Gerada a análise com suas devidas modificações nos ajustes de erro, o modelo proposto apresentou a seguinte razão: $\chi^{2} / \mathrm{gl}=1,25, \mathrm{GFI}=0,93 ; \mathrm{AGFI}=0,89 ; \mathrm{CFI}=0,96, \mathrm{NFI}=0,89, \mathrm{RMSEA}=0,05(0,03-0,07) ;$ o peso da variável do suporte organizacional associou-se positivamente ao capital psicológico no trabalho $(\lambda=0,55)$ e a expectativa de futuro $(\lambda=0,16)$, tendo, estas duas últimas variáveis, também, se relacionado positivamente $(\lambda=0,50)$. 
Ao verificar as saturações (Lambdas, $\lambda$ ), mesmo se encontrado dentro do intervalo esperado $\mid 0$ - 1|, na avaliação das estimativas de predição entre as associações de tais construtos (suporte organizacional, capital psicológico no trabalho e expectativa de futuro), à avaliação da preditividade entre as variáveis, a qual, tem por base a análise dos escores de regressão linear referente ao modelo teórico elaborado, observou-se, na associação entre o suporte organizacional e a expectativa de futuro, um resultado não significativo, estando acima de 0,05 (isto é, p < 0,18) e um escore Lambda abaixo de 0,30 (escore esse aceitável quando se pretende avaliar uma associação significativa). Com isso, rejeitou-se a hipótese inicial, a qual, havia proposto uma associação interdependente entre as três variáveis, condição que permitiu propor um modelo concorrente a ser verificado.

A partir dos resultados do modelo inicial, hipotetizou-se uma perspectiva mediacional entre as variáveis, questionando: haverá uma associação do suporte organizacional sobre a expectativa de futuro, porém, sendo esta influenciada pelo capital psicológico no trabalho? Com isso, foi efetuado o cálculo, com base em um modelo não recursivo, visando a verificação desta hipótese. Realizada as devidas modificações nos ajustes de erro, o pretenso modelo teórico revelou a seguinte razão estatística: $\chi^{2} / \mathrm{gl}=1,22, \mathrm{GFI}=0,95 ; \mathrm{AGFI}=0,91 ; \mathrm{CFI}=0,98, \mathrm{NFI}=0,98, \mathrm{RMSEA}=0,03(0,00-0,05)$.

Figura 1: Representação gráfica do modelo teórico alternativo hipotetizado.
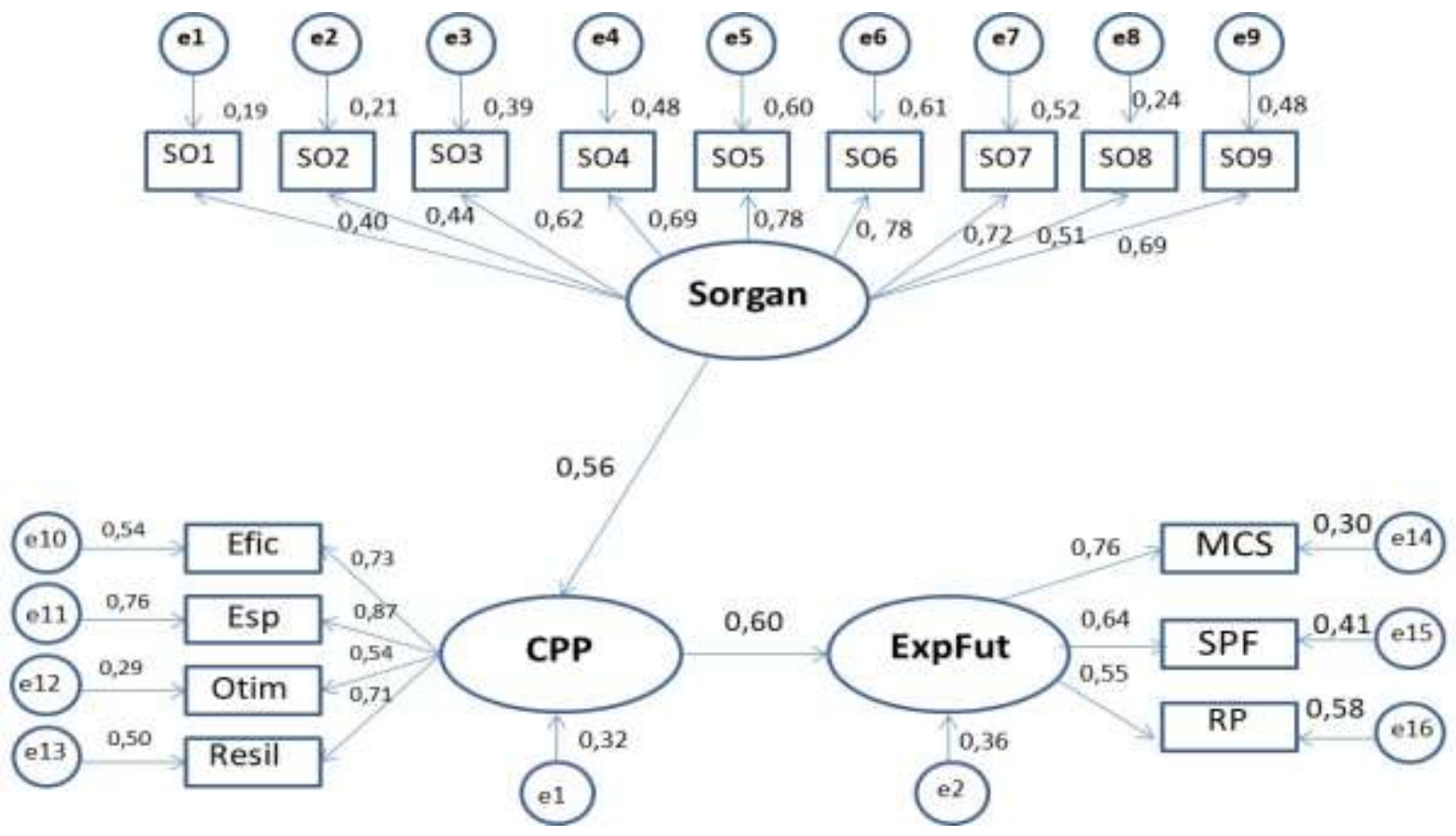

Notas: $\mathrm{CPP}=$ Capital Psicológico Positivo, Efic = Eficácia, Esp = Esperança, Otim = Otimismo, Resil = Resiliência, ExpFut $=$ Expectativa de Futuro, MCS = Melhores Condições da Sociedade, SPF = Sucesso Profissional e Financeiro, RP = Realização Pessoal, Sorgan = Suporte Organizacioal, SO1 = item 1 do Sorgan...SO9 = item 9 do Sorgan. Fonte: Autores.

Neste modelo concorrente, observou-se que o escore da variável do suporte organizacional associou-se, positivamente, ao capital psicológico no trabalho $(\lambda=0,56)$ e esta, à expectativa de futuro $(\lambda=0,59)$. Não apenas observaramse, que as saturações (Lambdas, $\lambda$ ) estiveram no intervalo $\mid 0$ - $1 \mid$, mas, também, ao consultar as estimativas de predição entre as associações das variáveis, todas foram significativas a um $\mathrm{p}<0,001$ (ver Tabela 1). Os indicadores psicométricos do modelo concorrente, além de ter sido melhor do que o hipotetizado inicialmente, sugere a segurança teórica do modelo mediacional se comparar a um modelo teórico sistêmico (no qual, todas as variáveis estão inter-relacionadas). 
Tabela 1: Indicadores das estimativas preditivas entre as variáveis do modelo.

\begin{tabular}{|c|c|c|c|c|c|c|}
\hline $\begin{array}{c}\text { Variáveis } \\
\text { (Itens/Fatores) }\end{array}$ & Relação & Construto & Estimativa & d.p. & $\begin{array}{l}\text { Razão } \\
\text { Crítica }\end{array}$ & p-valor $<$ \\
\hline $\begin{array}{c}\text { Capital } \\
\text { Psicológico } \\
\text { Positivo }\end{array}$ & $<---$ & Suporte Organizacional & 4,06 & 1,42 & 2,84 & 0,001 \\
\hline Expectativa de Futuro & $<---$ & Capital Psicológico Positivo & 0,43 & 0,11 & 3,88 & 0,001 \\
\hline SO01 & $<---$ & Suporte Organizacional & 1,00 & --- & --- & --- \\
\hline $\mathrm{SO} 02$ & $<---$ & Suporte Organizacional & 1,46 & 0,43 & 3,38 & 0,001 \\
\hline $\mathrm{SO} 03$ & $<---$ & Suporte Organizacional & 2,38 & 0,81 & 2,94 & 0,001 \\
\hline $\mathrm{SO} 04$ & $<---$ & Suporte Organizacional & 2,95 & 0,98 & 2,99 & 0,001 \\
\hline $\mathrm{SO} 05$ & $<---$ & Suporte Organizacional & 3,71 & 1,22 & 3,04 & 0,001 \\
\hline SO06 & $<---$ & Suporte Organizacional & 3,26 & 1,07 & 3,02 & 0,001 \\
\hline SO07 & $<---$ & Suporte Organizacional & 2,89 & 0,93 & 3,11 & 0,001 \\
\hline $\mathrm{SO} 08$ & $<---$ & Suporte Organizacional & 0,82 & 0,43 & 1,92 & 0,001 \\
\hline SO09 & $<---$ & Suporte Organizacional & 3,07 & 1,02 & 3,05 & 0,001 \\
\hline Resiliência & $<---$ & Capital Psicológico Positivo & 1,00 & --- & --- & --- \\
\hline Esperança & $<---$ & Capital Psicológico Positivo & 1,70 & 0,22 & 7,70 & 0,001 \\
\hline Autoeficácia & $<---$ & Capital Psicológico Positivo & 1,18 & 0,16 & 7,28 & 0,001 \\
\hline Otimismo & $<---$ & Capital Psicológico Positivo & 0,74 & 0,10 & 7,20 & 0,001 \\
\hline Realização pessoal & $<---$ & Expectativa de Futuro & 1,00 & --- & --- & --- \\
\hline Sucesso profissional & $<---$ & Expectativa de Futuro & 1,36 & 0,23 & 5,96 & 0,001 \\
\hline Melhores condições da sociedade & $<---$ & Expectativa de Futuro & 2,09 & 0,45 & 4,60 & 0,001 \\
\hline
\end{tabular}

Notas: SO01...SO09 = itens da escala de suporte organizacional; dp = Desvio Padrão; p-valor < 0,001. Fonte: Autores.

A fim de garantir a consistência dos resultados observados no modelo teórico proposto, procurou também, avaliar o efeito da variável mediacional (isto é, o capital psicológico positivo), para isso, efetuou o teste de Sobel (z), no qual, pode-se verificar a influência entre as variáveis dependente sobre a independente a partir do nível de mediação direta e indireta entre elas (Baron, \& Kenny, 1986). De acordo com os autores supracitados, a variável mediadora (VMe) é uma terceira variável que deve predizer a variável dependente (VD), com a variável independente (VI) sendo preditora da variável mediadora. Assim, na presença de ambas - VI e VMe - uma relação significativa prévia entre a VI e a VD decresce em magnitude, demonstrando o efeito mediacional. Com isso, na Figura 2, pode-se observar um modelo mediacional, o qual sugere que a suporte organizacional (VI) influenciou, positivamente, o capital psicológico positivo (VMe), tendo esta, influenciado a expectativa de futuro (VD) $(\mathrm{z}=7,72 ; \mathrm{p}<0,001)$. 
Figura 2: Modelo mediacional entre Suporte organizacional e expectativa de futuro, por meio capital psicológico positivo.

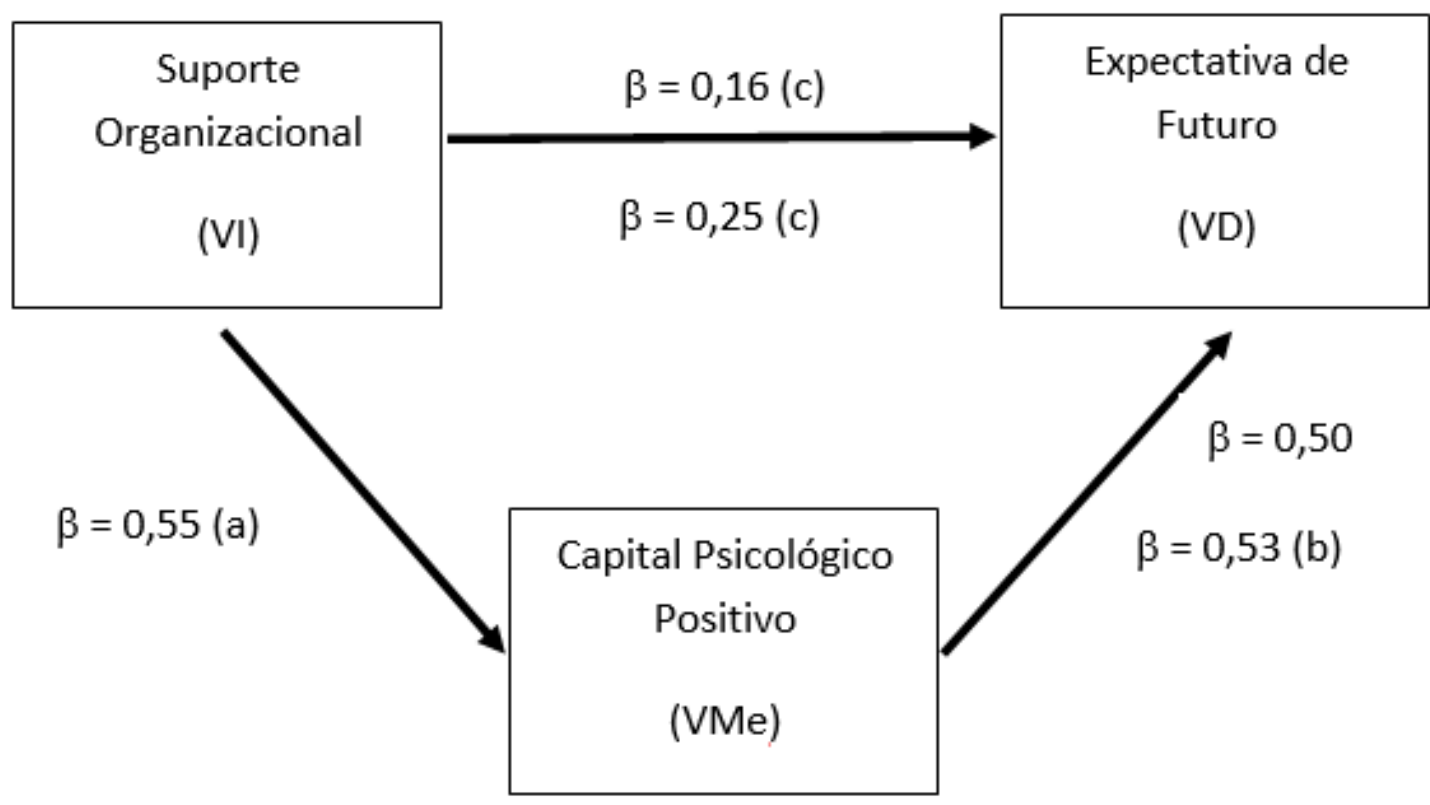

Fonte: Autores.

Com isso, o suporte organizacional influência muito mais a expectativa de futuro quando é mediado pelo capital psicológico positivo; observou-se que o efeito preditivo é maior a partir do caminho explicativo indireto $\left(R^{2}=0,25\right)$ do que do caminho direto $\left(R^{2}=0,16\right)$. Na Figura 1 , pode-se observar que, no caminho $a \rightarrow b$, com VMe, o Beta $(\beta)$ além de maior no caminho 'c' através da mediação.

\section{Discussão}

De forma geral, as influências entre as variáveis no modelo teórico proposto, sugere novos espaços da prática do recurso humano nas organizações, especialmente, no que se refere as práticas que saliente uma perspectiva mais positiva do comportamento organizacional destinadas ao estímulo e manutenção da satisfação e saúde do trabalhador. Destaca-se a confirmação da hipótese alternativa, na qual, o suporte organizacional tem melhor poder explicativo sobre a expectativa de futuro quando mediado pelo capital psicológico positivo; isto é, não é possível unilateralizar a organização como a única responsável pelos fatores de proteção dos seus funcionários na estrutura da saúde emocional deste, é preciso fazer refletir no próprio funcionário, que ele é parte responsabilidade de si mesmo (cf. Marujo, Neto, \& Rivero, 2007; Brandão, BorgesAndrade, \& Guimarães, 2012; Boehs \& Silva, 2017; Formiga \& Guimarães, 2019).

Faz-se necessário associar forças (organização e funcionários) para que ambos possam ter êxitos em sua produtividade e manutenção da saúde psíquica e organizacional (Csikszentmihalyi, 2004; Cavalcante, Siqueira, \& Kuniyoshi, 2014). Com o modelo alternativo corroborado, principalmente, quando este foi comparado aos indicadores preditivos observados no modelo original (o qual, hipotetizado inicialmente), os resultados não apenas foram significativos, mas, os indicadores tidoS como essenciais para confirmar o modelo [por exemplo, $\chi^{2} / \mathrm{gl}$, GFI, AGFI, CFI, NFI e RMSEA (cf. Hair, Tatham, Anderson, \& Black, 2005)] foram bem melhores. a adequação teórico-empírica que se propôs e que poderá ser aplicada na dinâmica organizacional para alinhar, junto aos recursos humanos, práticas organizacionais de ajuste emocionais dos funcionários.

As reflexões expostas acima, podem ser destacadas ao observar os resultados apresentados na Figura 2; a partir dela, procurou-se verificar a indicação do modelo teórico observado, na qual, revelou um aumento no escore relacional entre 
suporte organizacional e expectativa de futuro, quando o capital psicológico mediou essas variáveis. As associações entre as variáveis, não poderão ser pensadas como exclusivas do problema organizacional, mas, também, sugere um melhor desenvolvimento por parte do funcionário da empresa, especialmente, em relação aos fatores psicológicos organizacionais na vida de trabalho.

Tais reflexões, associadas aos resultados, convergem com os estudos de Cunha, Rego e Lopes (2013) e Gonçalves (2016) em relação ao comportamento organizacional positivo; para esses autores, a necessidade de uma perspectiva organizacional com foco na abordagem humanista e positiva da ciência psicologia aplicada é cada vez mais significativa, não apenas visando um prazer do trabalhar, mas, também, o surgimento e manutenção de um clima positivo no ambiente de trabalho.

Sendo assim, a expectativa em um futuro melhor, na perspectiva do empregado, tomando como base de orientação para essa reflexão, ainda o modelo mediacional, não pode ser considerado responsabilidade única da organização mas, também é preciso fomentar interesse e responsabilidade do próprio profissional; afinal, mais do que o sistema social e econômico em que o sujeito estar incluso, a maior motivação para ascender em todo esse sistema é do próprio trabalhador, o qual, desenvolve mecanismos de defesa e ferramentas emocionais e funcionais justas para que seus objetivos sejam estabelecidos, consequentemente, mantidos ao longo do seu desenvolvimento laboral.

Ao refletir nesta direção, apoia-se justamente, nas concepções de Fadiño, Fomiga, Menezes (2018) referente a importância e exigência de atitudes inovadores dos profissionais, para os quais, leva-se em conta fatores intrínsecos e extrínsecos; enquanto o primeiro trata-se de uma adesão do papel socio-organizacional do trabalhador, o segundo seria do interesse (e necessidade) da organização, ambos trabalhando sob via de mão dupla quanto a formação trabalhador-culturaorganização para o comportamento social e organizacionalmente desejável da produtividade, mesmo que pudessem gerar elementos de proteção de forma específica em cada variável-contexto (isto é, indivíduo e organização), faz-se necessário compartilharem entre eles como objetivo de responder demandas de Recursos Humanos para qualificação trabalhadororganização.

\section{Considerações Finais}

Espera-se que os objetivos tenham sidos cumpridos e mesmo tendo corroborado a hipótese alternativa pretendida, é preciso destacar que o foco central da pesquisa pauta-se na contribuição da explicação do desenvolvimento psicológico do trabalhador na organização, principalmente, na apresentação de elementos de um recurso humano mais humanista e que aponte em direção de variáveis preditivas relacionadas a dinâmica de uma estrutural e funcionalidade do ajustamento emocional nos pilares funcionário-comportamento organizacional-organização destinado a produtividade no trabalho.

As associações observadas no modelo teórico mediacional estabelecido destaca que, para um desenvolvimento mais satisfatório na organização e no trabalhador, é preciso contemplar um investimento emocional (isto é, um capital psicológico) concentrando mais na qualidade da manutenção das emoções do que a própria cultura e políticas organizacionais. Seria muito útil, na área do recurso humano, uma agenda de pesquisa e intervenção na área da psicologia organizacional e do trabalho, salientando mais aspectos os saudáveis do trabalhador. Mesmo com resultados adequados ao objetivo do estudo, é preciso destacar alguns limites para futuras pesquisas: Seria de importante a construção e testagem de um modelo teórico que avalie a associação das mesmas variáveis empresas da zona urbana e rural no contexto de pesquisa; também, poderia associar esse modelo teórico com a variável conflito família-trabalho e trabalho-família verificando extensão organização-vida pessoal; e por fim, não menos importante, deve-se buscar realizar um estudo de replicação do modelo observado neste artigo a fim de verificar a invariância métrica e associativa entre as variáveis. 


\section{Referências}

Ahmad, I., \& Zafar, M. A. (2018). Impact of psychological contract fulfillment on organizational citizenship behavior: Mediating role of perceived organizational support. International Journal of Contemporary Hospitality Management, 30(2), 1001-1015.

Akgunduz, Y., Alkan, C., \& Gök, Ö. A. (2018). Perceived organizational support, employee creativity and proactive personality: the mediating effect of meaning of work. Journal of Hospitality and Tourism Management, 34, 105-114.

Al-Hussami, M., Hammad, S., \& Alsoleihat, F. (2018). The influence of leadership behavior, organizational commitment, organizational support, subjective career success on organizational readiness for change in healthcare organizations. Leadership in Health Services.

Altinoz, M., Cop, S., Cakiroglu, D., \& Altinoz, O. T. (2016). The Influence of Organization Support Perceived in Enterprises on Burnout Feeling: A Field Research. Procedia-Social and Behavioral Sciences, 235, 427-434.

Anglin, A. H., Short, J. C., Drover, W., Stevenson, R. M., McKenny, A. F., \& Allison, T. H. (2018). The power of positivity? The influence of positive psychological capital language on crowdfunding performance. Journal of Business Venturing.

Ascenção, C. (2009). Práticas de Gestão de Carreira, Acolhimento e Integração e Empenhamento Organizacional: Estudo de Caso no sector do Pós-Venda Automóvel. (Dissertação de Mestrado). Universidade de Lisboa, Lisboa, Portugal.

Aspinwall, L. G. (2006) The Psychology of Future-Oriented Thinking: From Achievement to Proactive Coping, Adaptation, and Aging. Motivation and Emotion, 29(4), 203-235.

Avey, J. B., Hughes, L. W., Norman, S. M., \& Luthans, K. W. (2008a). Using positivity, transformational leadership and empowe rment to combat employee negativity. Leadership \& Organization Development Journal, 29(2), 110-126.

Avey, J. B., Luthans, F., \& Jensen, S. M. (2009). Psychological capital: A positive resource for combating employee stress and turnover. Human resource management, 48(5), 677-693.

Avey, J. B., Luthans, F., \& Mhatre, K. H. (2008b). A call for longitudinal research in positive organizational behavior. Journal of Organizational Behavior: The International Journal of Industrial, Occupational and Organizational Psychology and Behavior, 29(5), $705-711$.

Avey, J. B., Patera, J. L., \& West, B. J. (2006). The implications of positive psychological capital on employee absenteeism. Journal of Leadership \& Organizational Studies, 13(2), 42-60.

Badran, M. A., \& Youssef-Morgan, C. M. (2015). Psychological capital and job satisfaction in Egypt. Journal of Managerial Psychology, 30(3), 354-370.

Brandão, H. P., Borges-Andrade, J. E., \& Guimarães, T. A. (2012). Desempenho organizacional e suas relações com competências gerenciais, suporte organizacional e treinamento. Revista de Administração da USP, 47(4), 523-539. 10.5700/rausp1056.

Burmeister, A., \& Deller, J. (2016). A practical perspective on repatriate knowledge transfer: The influence of organizational support practices. Journal of global mobility, 4(1), 68-87.

Buscacio, R. C. Z., \& Soares, A. B. (2017). Expectativas sobre o desenvolvimento da carreira em estudantes universitários. Revista Brasileira de Orientação Profissional, 18(1), 69-79.

Byrne, Z. S., \& Hochwarter, W. A. (2008). Perceived organizational support and performance: Relationships across levels of organizational cynicism. Journal of Managerial Psychology, 23(1), 54-72.

Cárdenas, M. T. F., Loving, R. D., \& Lagunes, I. (2011). Desarrollo y Validación de una Escala para Medir Apoyo Social en Adultos con Perspectiva de Apego. Revista Iberoamerica de Diagnóstico y Evaluación Psicológica, 32(2), 9- 34.

Cavalcante, M. M., Siqueira, M. M. M., \& Kuniyoshi, M. S. (2014). Engajamento, bem estar no trabalho e capital psicológico: um estudo com profissionais da área de gestão de pessoas. Pensamento \& Realidade, 29(4), 42-64.

Celep, C., \& Yilmazturk, O. E. (2012). The relationship among organizational trust, multidimensional organizational commitment and perceived organizational support in educational organizations. Procedia-Social and Behavioral Sciences, 46, 5763-5776.

Chiu, W. C. (2002). Do types of economic ownership matter in getting employees to commit? An exploratory study in the People's Republic of China. International Journal of Human Resource Management, 13(6), 865-882.

Chiu, W. C., Harry Hui, C., \& Lai, G. W. (2007). Psychological ownership and organizational optimism amid China's corporate transformation: Effects of an employee ownership scheme and a management-dominated board. The International Journal of Human Resource Management, 18(2), 303-320.

Choi, Y., \& Lee, D. (2014). Psychological capital, Big Five traits, and employee outcomes. Journal of Managerial Psychology, 29(2), 122-140.

Chong, H., White, R. E., \& Prybutok, V. (2001). Relationship among organizational support, JIT implementation, and performance. Industrial Management e data systems, 101(6), 273-280.

Cooper-Hakim, A., \& Viswesvaran, C. (2005). The construct of work commitment: testing an integrative framework. Psychological Bulletin, 131 (2), 241259.

Costa, B. S., Costa, S. D. S., \& Cintra, C. L. D. (2018). Os possíveis impactos da reforma da legislação trabalhista na saúde do trabalhador. Rev. Bras. Med. Trab, 16(1), 109-117.

Csikszentmihalyi, M. (2004). Gestão qualificada: a conexão entre felicidade negócio. Bookman. 
Cunha, M. P., Rego, A., \& Lopes, M. P. (2013). Comportamento organizacional positivo. Análise Psicológica, 31(4), $313-328$.

Dawley, D. D., Andrews, M. C., \& Bucklew, N. S. (2008). Mentoring, supervisor support, and perceived organizational support: what matters most?. Leadership \& Organization Development Journal, 29(3), 235-247.

Drucker, P. F. (1959). Long-range planning — challenge to management science. Management science, 5(3), 238-249.

Eisenberger, R. et al. (1986). Perceived organizational support. Journal of Applied Psychhology, 72(3), 500-507.

Fernandes, C. M., Siqueira, M. M. M., \& Vieira, A. M. (2014). Impacto da percepção de suporte organizacional sobre o comprome timento organizacional afetivo: o papel moderador da liderança. Revista Pensamento Contemporâneo em Administração, 8(4), $140-162$.

Ferreira, A. I., Cardoso, C., \& Braun, T. (2018) "The mediating effects of ego-resilience in the relationship between organizational support and resistance to change", Baltic Journal of Management, Vol. 13(1), 104-124, https://doi.org/10.1108/BJM-06-2017-0171

Fleury, L. F. O., Formiga, N. S., Souza, M. A., \& Souza, M. A. F. (2017). Escala de Percepção de Suporte Organizacional: evidência da estrutura fatorial em trabalhadores brasileiros. Psicologia em Pesquisa, 11(1), 1-2. https://dx.doi.org/10.24879/201700110010033

Ford, C. M. (2002). The futurity of decisions as a facilitator of organizational creativity and change. Journal of Organizational Change Management, 15(6), $635-646$.

Formiga, N. S., \& Souza, M. A. D. (2014). Comprovação empírica de uma medida psicológica sobre a percepção do suporte organizacional em trabalhadores de diferentes empresas. Boletim-academia paulista de psicologia, 34(87), 510-552.

Formiga, N. S., Fleury, L. F. O., \& Souza, M. A. (2015). Evidência psicométrica da versão reduzida da escala de expectativa de futuro. Revista de Psicologia, 6(1).

Formiga, N. S., Freire, B. G. O., Batista, P. F. A., \& Estevam, I. D. (2017). Suporte organizacional e autoestima em fun cionários de organizações públicas e privadas no Brasil. Psicologia PT, 1-15.

Formiga, N. S., Freire, B. G. O., Batista, P. F. A., \& Estevam, I. D. (2017). Suporte organizacional e autoestima em funcionários de organizações públicas e privadas no brasil. Recuperado em 30 Julho de 2018, http://www.psicologia.pt/artigos/ver_artigo.php?suporte-organizacional-e-autoestima-em-funcionariosde-organizacoes-publicas-e-privadas-no-brasil\&codigo $=\mathrm{A} 1142 \&$ area $=\mathrm{d} 8$

Formiga, N. S., \& Guimaraes, W. N. C. (2019). Caminhos para a rentabilidade emocional da saúde laboral: A influência do capital psicológico positivo no transtorno emocional em médicos. Beau Bassin - Mauritius: Nova edições acadêmicas.

Gonçalves, J. R. D. (2016). Climas organizacionais positivos, relação ótima em equipa e compromisso organizacional. (Tese de doutorado). Universidade Autónoma de Lisboa, Lisboa, Portugal.

Graso, M., \& Probst, T. M. (2012). The Effect of Consideration of Future Consequences on Quality and Quantity Aspects of Job Performance. Journal of Applied Social Psychology, 42(6), 1335-1352.

Günther, I. A., \& Günther, H. (1998). Brasílias pobres, Brasílias ricas: perspectivas de futuro entre adolescentes. Psicologia: Reflexão e Crítica, 11(2), 191207.

Han, K. S., \& Garg, P. (2018). Workplace democracy and psychological capital: a paradigm shift in workplace. Management Research Review.

Han, Y., Brooks, I., Kakabadse, N. K., Peng, Z., \& Zhu, Y. (2012). A grounded investigation of Chinese employees' psychological capital. Journal of Managerial Psychology, 27(7), 669-695.

Hubbard, N., \& Purcell, J. (2001). Managing employee expectations during acquisitions. Human Resource Management Journal, 11(2), 17-33.

Ibrahim, H. I., Isa, A., \& Shahbudin, A. S. M. (2016). Organizational support and creativity: The role of developmental experiences as a moderator. Procedia Economics and Finance, 35, 509-514.

Ibrahim, M. E., \& Al Marri, A. (2015). Role of gender and organizational support in work-family conflict for accountants in UAE. International Journal of Commerce and Management, 25(2), 157-172.

Islam, T., Ali, G., \& Ahmed, I. (2018). Protecting healthcare through organizational support to reduce turn over intention. International Journal of Human Rights in Healthcare, 11(1), 4-12.

Jawahar, I. M., \& Hemmasi, P. (2006). Perceived organizational support for women's advancement and turnover intentions: The mediating role of job and employer satisfaction. Women in Management Review, 21(8), 643-661.

Joireman, J., Daniels, D., George-Falvy, J., \& Kamdar, D. (2006). Organizational citizenship behaviors as a function of empathy, consideration of future consequences, and employee time horizon: An initial exploration using na in-basket simulation of OCBs. Journal of Applied Social Psychology, 39, 22662292 .

Kang, H. J. A., \& Busser, J. A. (2018). Impact of service climate and psychological capital on employee engagement: The role of organizational hierarchy. International Journal of Hospitality Management, 75, 1-9.

Karatepe, O. M., \& Karadas, G. (2015). Do psychological capital and work engagement foster frontline employees' satisfaction? A study in the hotel industry. International Journal of Contemporary Hospitality Management, 27(6), 1254-1278.

Lee, J., Yun, S., \& Kim, S. L. (2017). Consideration of future consequence and task performance: The moderating effects of support. Journal of Managerial Psychology, 32(7), 497-512. 
Li, Y., Castaño, G., \& Li, Y. (2018). Linking leadership styles to work engagement: The role of psychological capital among Chinese knowledge workers. Chinese Management Studies, 12(2), 433-452.

Linley, P., Govindji, R., \& West, M. (2007). Positive psychology approaches to public services leadership: An introduction to strengths-based leadership. International Journal of Leadership in Public Services, 3(4), 44-55.

Luthans, F. (2002). The need for and meaning of positive organizational behavior. Journal of Organizational Behavior, 23(6), 695-706.

Luthans, F., Avolio, B. J., Avey, J. B., \& Norman, S. M. (2007). Positive psychological capital: Measurement and relationship with performance and satisfaction. Personnel Psychology, 60(3), 541-572.

Luthans, F., Youssef, C. M., \& Avolio, B. J. (2007). Psychological Capital: Developing the human competitive edge. Oxford University Press.

Margolis, S. L., \& Hansen, C. D. (2003). Visions to guide performance: a typology of multiple future organizational images. Performance Improvement Quarterly, 16(4), 40-58.

Martinez, M. C., \& Paraguay, A. I. B. B. (2003). Satisfação e saúde no trabalho: aspectos conceituais e metodológicos. Cadernos de Psicologia Social do Trabalho, 6, 59-78. http://pepsic.bvsalud.org/scielo.php?script=sci_arttext\&pid=S1516-37172003000200005\&lng=pt\&tln g=pt.

Menezes, I. G., \& Gomes, A. C. P. (2010). Clima organizacional: Uma revisão histórica do construto. Psicologia em Revista, 6(1), 158-179.

Moreno, B. S. (2002). Gestão de pessoas: Tendências e desafios na nova missão do RH de hoje. Revista de Ciências Jurídicas, 3(2).

Mossholder, K. W., Settoon, R. P., Harris, S. G., \& Armenakis, A. A. (1995). Measuring emotion in open-ended survey responses: An application of textual data analysis. Journal of management, 21(2), 335-355.

Ng, E. S., \& Parry, E. (2016). Multigenerational research in human resource management. In Research in Personnel and Human Resources Management (pp. 1-41). Emerald Group Publishing Limited.

Ng, K. H. (2017). The fundamental role of social support in cultivating motivation to improve work through learning. Industrial and Commercial Training, 49(1), 55-60.

Oliveira-Castro, G. A. D., Pilati, R., \& Borges-Andrade, J. E. (1999). Percepção de suporte organizacional: desenvolvimento e validação de um questionário. Revista de Administração Contemporânea, 3(2), 29-51.

Omar, A., Delgado, H. U., Souza, M. A., \& Formiga, N. S. (2005). Perspectivas de futuro y búsqueda de sensaciones en jóvenes estudiantes: Un estudio entre Brasil y Argentina. Revista Latinoamericana de Estudios Educativos, 35(1-2), 165-180.

Ouweneel, E., Le Blanc, P. M., \& Schaufeli, W. B. (2013). Do-it-yourself: An online positive psychology intervention to promote positive emotions, selfefficacy, and engagement at work. Career Development International, 18(2), 173-195.

Paschoal, T. (2008). Bem-estar no trabalho: relações com suporte organizacional, prioridades axiológicas e oportunidades de alcance de valores pessoais no trabalho (Tese de doutorado). Universidade de Brasília, Brasília, DF, Brasil.

Paschoal, T. (2008). Bem-estar no trabalho: relações com suporte organizacional, prioridades axiológicas e oportunidades de alcance de valores pessoais no trabalho (Tese de doutorado). Universidade de Brasília, Brasília, DF, Brasil.

Paschoal, T., Torres, C. V., \& Porto, J. B. (2010). Felicidade no trabalho: relações com suporte organizacional e suporte social. Revista de Administração Contemporânea, 14(6), 1054-1072. https://dx.doi.org/10.1590/S1415-65552010000700005

Pereira A.S. et al. (2018). Metodologia da pesquisa científica. UFSM.

Puente-Palacios, K., \& Peixoto, A. L. A. (2015). Ferramentas de diagnóstico para organizações e trabalho: um olhar a partir da psicologia: Artmed.

Rehman, S. U., Qingren, C., Latif, Y., \& Iqbal, P. (2017). Impact of psychological capital on occupational burnout and performance of faculty members. International Journal of Educational Management, 31(4), 455-469

Richardson, H. A., Yang, J., Vandenberg, R. J., DeJoy, D. M., \& Wilson, M. G. (2008). Perceived organizational support's role in stressor-strain relationships. Journal of Managerial Psychology, 23(7), 789-810.

Robbins, R. N. \& Bryan, A. (2004). Relationships Between Future Orientation, Impulsive Sensation Seeking, and Risk Behavior Among Adjudicated Adolescents. Journal of Adolescent Research, 19(4), p. 428-445.

Schmidt, C. M. (2008). Construcción de un cuestionario de emociones positivas en población entrerriana. Revista Iberoamericana de Diagnóstico y Evaluación Psicológica, 26(2), 117-139.

Scorsolini-Comin, F., Forli Inocente, D., \& Kazumi Miura, I. (2012). Avaliação de um programa de treinamento corporativo: Em busca da mudança organizacional. Revista Brasileira de Orientação Profissional, 13(1).

Seginer, R. (2009). Future orientation: Developmental and ecological perspectives. Springer.

Seligman, M. E. P., \& Csikszentmihalyi, M. (2000). Positive Psychology: An introduction. American Psychologist, 55(1), 5-14. 10.1037/0003-066X.55.1.5

Silva E. E. C., \& Bastos, A. V. B. (2010). A escala de consentimento organizacional: construção e evidências de sua validade. Revista Psicologia: Organizações e Trabalho, 10(1), 7-22. 
Singhal, H., \& Rastogi, R. (2018). Psychological capital and career commitment: the mediating effect of subjective well-being. Management Decision, 56(2), 458-473.

Siqueira, M. M. M. (2009). Medidas do comportamento organizacional: ferramentas de diagnóstico e de gestão. Artmed Editora.

Souza, M. A. D., Pereira, P. R. F., Funck, A. L., \& Formiga, N. S. (2013). Consistência interna e estrutura fatorial da escala de expectativa de futuro em brasileiros. Boletim-Academia Paulista de Psicologia, 33(85), 330-353.

Souza, M., Pereira, P., Funck, A., \& Formiga, N. (2013). Consistência interna e estrutura fatorial da escala de expectativa de futuro em brasileiros. Boletim da Academia Paulista de Psicologia, 33(85), 330-353.

Ströbel, T., Maier, C., \& Woratschek, H. (2018). How to reduce turnover intention in team sports? Effect of organizational support on turnover intention of professional team sports athletes. Sport, Business and Management: An International Journal, 8(2), 98-117.

Suchman, A. L., Sluyter, D. J., \& Williamson, P. R. (Eds.). (2011). Leading change in healthcare: transforming organizations using complexity, positive psychology and relationship-centered care. Radcliffe Publishing.

Sulimani-Aidan, Y., \& Benbenishty, R. (2011) Future expectations of adolescents in residential care in Israel. Children and Youth Services Review, 33 (7), $1134-1141$.

Tamayo, A., \& Paschoal, T. (2003). A relação da motivação para o trabalho com as metas do trabalhador. Revista de Administração Contemporânea, 7(4), 33-54.

Tamayo, N., \& Abbad, G. D. S. (2006). Autoconceito profissional e suporte à transferência e impacto do treinamento no trabalho. Revista de Administração Contemporânea, 10(3), 9-28.

Tolfo, S. D. R., \& Piccinini, V. C. (2007). Sentidos e significados do trabalho: explorando conceitos, variáveis e estudos empíricos brasileiros. Psicologia \& Sociedade, 19(1)

Tractenberg, L. (1999). A complexidade nas organizações: futuros desafios para o psicólogo frente à reestruturação competitiva. Psicologia: ciência e profissão,19(1), 14-29.

Tüzün, I. K., Çetin, F., \& Basim, H. N. (2018). Improving job performance through identification and psychological capital. International Journal of Productivity and Performance Management, 67(1), 155-170.;

Ujhelyi, K., Carson, J., \& Holland, M. (2016). Positive psychology in dual diagnosis: a preliminary investigation. Advances in Dual Diagnosis, 9(4), 139-153.

Vasconcellos, V. C. D. (2015). Antecedentes e consequentes de expectativas de carreira e de futuro organizacional. Tese de Doutorado. Universidade de Brasília.

Vasconcellos, V. C. D., \& Neiva, E. R. (2017). Escala de Expectativas de Futuro Organizacional: Desenvolvimento e Evidências de Validade. REAd. Revista Eletrônica de Administração (Porto Alegre), 23(2), 58-82.

Verstegen, B. H. (2011). A socio-economic view on management control. International journal of social economics, 38(2), 114-127.

Weick, K. E. (1979). The social psychology of organizing (Topics in social psychology series). Columbus, OH: McGraw-Hill Humanities.

Wu, C. M., \& Chen, T. J. (2018). Collective psychological capital: Linking shared leadership, organizational commitment, and creativity. International Journal of Hospitality Management, 74, 75-84.

Zanelli, J. C., Borges-Andrade, J. E., \& Bastos, A. V. B. (2014). Psicologia, Organizações e Trabalho no Brasil-2. AMGH Editora.

Zhou, K. Z., Li, J. J., \& Zhou, N. (2004). Employee's perceptions of market orientation in a transitional economy: China as an example. Journal of Global Marketing, 17(4), 5-22. 